\title{
Prognostic value of mitotic checkpoint protein BUB3, cyclin B1, and pituitary tumor-transforming 1 expression in prostate cancer
}

 \\ Tarjei S. Hveem ${ }^{1} \cdot$ Hanne A. Askautrud ${ }^{1} \cdot$ Marco Novelli $^{1,4} \cdot$ Håkon Wæhre $^{1} \cdot$ Knut Liestøl $^{1,3} \cdot$ Håvard E. Danielsen $^{1,3,5}$
}

Received: 19 July 2019 / Revised: 21 October 2019 / Accepted: 31 October 2019 / Published online: 4 December 2019

(c) The Author(s) 2019. This article is published with open access

\begin{abstract}
The mitotic checkpoint protein BUB3, cyclin B1 (CCNB1) and pituitary tumor-transforming 1 (PTTG1) regulates cell division, and are sparsely studied in prostate cancer. Deregulation of these genes can lead to genomic instability, a characteristic of more aggressive tumors. We aimed to determine the expression levels of BUB3, CCNB1, and PTTG1 as potential prognostic markers of recurrence after radical prostatectomy. Protein levels were determined by immunohistochemistry on three formalin-fixed paraffin-embedded tissue sections from each of the 253 patients treated with radical prostatectomy. Immunohistochemistry scores were obtained by automated image analysis for CCNB1 and PTTG1. Recurrence, defined as locoregional recurrence, distant metastasis or death from prostate cancer, was used as endpoint for survival analysis. Tumors having both positive and negative tumor areas for cytoplasmic BUB3 (30\%), CCNB1 (28\%), or PTTG1 (35\%) were considered heterogeneous. Patients with $\geq 1$ positive tumor area had significantly increased risk of disease recurrence in univariable analysis compared with patients where all tumor areas were negative for cytoplasmic BUB3 (hazard ratio $[\mathrm{HR}]=2.18,95 \%$ confidence interval $[\mathrm{CI}] 1.41-3.36$ ), CCNB1 (HR $=2.98,95 \%$ CI 1.93-4.61) and PTTG1 $(\mathrm{HR}=1.91,95 \%$ CI 1.23-2.97). Combining the scores of cytoplasmic BUB3 and CCNB1 improved risk stratification when integrated with the Cancer of the Prostate Risk Assessment post-Surgical (CAPRA-S) score (difference in concordance index $=0.024,95 \% \mathrm{CI} 0.001-0.05$ ). In analysis of multiple tumor areas, prognostic value was observed for cytoplasmic BUB3, CCNB1, and PTTG1.
\end{abstract}

Supplementary information The online version of this article (https:// doi.org/10.1038/s41379-019-0418-2) contains supplementary material, which is available to authorized users.

Håvard E. Danielsen

hdaniels@labmed.uio.no

1 Institute for Cancer Genetics and Informatics, Oslo University Hospital, NO-0424 Oslo, Norway

2 Department of Pathology, Oslo University Hospital, NO-0424 Oslo, Norway

3 Department of Informatics, University of Oslo, NO-0316 Oslo, Norway

4 Research Department of Pathology, University College London Medical School, London WX1E 6DE, UK

5 Nuffield Division of Clinical Laboratory Sciences, University of Oxford, Oxford OX3 9DU, UK

\section{Introduction}

Intra-tumor heterogeneity is common in prostate cancer, and may to some extent explain the difficulties in establishing useful molecular markers for this disease [1]. Intra-tumor heterogeneity is a beneficial trait for cancer progression and it likely develops due to genomic instability [2], which can be induced by defects in the mechanisms that regulate mitosis. The mitotic checkpoint controls mitosis through the mitotic checkpoint complex, where the mitotic checkpoint protein BUB3 is a key component [3]. Correct attachment of the kinetochores inactivates the mitotic checkpoint complex, which facilitates the degradation of cyclin B1 (CCNB1) and pituitary tumor-transforming 1 (PTTG1), and determines exit from mitosis [3].

Defects in the mitotic checkpoint may lead to chromosome mis-segregation, generating aneuploidy which is a marker of poor prognosis in many cancer types $[4,5]$. The impairment in function of the mitotic checkpoint is often associated with changes in the levels of proteins involved in 
the checkpoint [4]. The expression of BUB3, CCNB1, and PTTG1 has been shown to correlate with tumor grade and prognosis in some cancers [6-8]. However, their prognostic role in prostate cancer is unclear.

As better risk stratification of patients with prostate cancer is needed [9], we aimed to investigate whether the expression of BUB3, CCNB1 and PTTG1 could independently predict recurrence after radical prostatectomy. Protein levels were assessed by immunohistochemistry and performed on three tumor-containing tissue sections for each patient $(n=253)$ in order to account for intra-tumor heterogeneity. The mRNA counts were determined in one tumor-containing tissue sample for each patient.

\section{Materials and methods}

\section{Patients and specimens}

A cohort of 317 patients with primary prostate cancer was treated with radical prostatectomy at a tertiary comprehensive cancer center in Norway between 1987 and 2005 (The Norwegian Radium Hospital, Oslo). The basis of prostatectomy was preoperative absence of known metastases, age $<75$ years and life expectancy of $\geq 10$ years. Neoadjuvant therapy was not given to any of the patients included in the analyses. Radiation and androgen deprivation were only given after indication of recurrence. This study adhered to the REporting recommendations for tumor MARKer prognostic studies reporting criteria [10] and was approved by the Regional Committees for Medical and Health Research Ethics (REK), Norway (REK no. S-07443a). All tissue sections with tumors were Gleason graded by an experienced uropathologist (LV) according to the updated 2005 International Society of Urological Pathology Consensus guidelines [11]. Three tumor-containing tissue blocks were selected based on the highest Gleason score and/or previously reported worst DNA ploidy status [1]. Sufficient tumor material for analyses was available for 253 patients (Fig. 1). Tumor areas that measured $<4 \mathrm{~mm}^{2}$ on a diagnostic hematoxylin and eosin tissue section or immune stained sections were excluded. Tumor areas within one section were analyzed separately if they were situated $\geq 3 \mathrm{~mm}$ apart. Two to four tumor areas were found on 104 of the 759 included blocks.

\section{Immunohistochemistry}

Three micrometers thick tissue sections were cut and mounted on Superfrost plus slides (Thermo Scientific, Waltham, MA), dried at $60^{\circ} \mathrm{C}$ and stored at $-80^{\circ} \mathrm{C}$. The Envison FLEX + system/Dako Autostainer Link 48 (Agilent Technologies, Santa Clara, CA) was used for the immunohistochemistry procedure. Parallel tissue sections were incubated with antibodies against BUB3, CCNB1, and PTTG1 (Supplementary Table 1). The specificity of the BUB3 antibody was confirmed with the Human Bub3 peptide (Abcam, Cambridge, UK), following the manufacturer's recommendations. Positive and negative controls were included in each run. All sections were counterstained with hematoxylin and scanned at $\times 40$ (NanoZoomer HT, Hamamatsu Photonics, Hamamatsu, Japan). Tumor areas with $\geq 95 \%$ of the tumor tissue lost during processing were excluded (301 of 2277 tissue sections, 13\%, Fig. 1).

\section{Visual scoring of BUB3}

The complete tumor areas were evaluated using NDP. view2 (Hamamatsu Photonics, Hamamatsu, Japan) at $\times 10$ magnification (EE). Pre-defined thresholds were used to categorize the scores. As there is no consensus on how to score BUB3, the thresholds were selected after a visual examination of the staining pattern of BUB3. Nuclear BUB3 was normally expressed in all tumor cells and a tumor area was considered positive when $\geq 99 \%$ of the tumor cells had nuclear BUB3. Cytoplasmic expression was observed in single cells scattered throughout the tumor area, and were commonly observed in mitotic cells. A cut-off value of $>5 \%$ was chosen so that a sample was only considered positive when the levels of cytoplasmic BUB3 exceeded the background levels of positivity. Cytoplasmic BUB3 was also scored independently in $1 / 3$ of the tissue sections by a pathologist (MP). BUB3 could not be scored automatically due to overlapping color spectra between positive cytoplasm and nuclei. In addition, a less accurate count was obtained for poorly differentiated tumors, as the accuracy of the count was dependent on the degree of clustering of cells.

\section{Automated scoring of CCNB1 and PTTG1}

The complete tumor areas were marked manually in ImmunoPath (Room4 Group Ltd, Crowborough, UK), while avoiding artifacts, lymphocytes, and intermixed benign glands. Tiles that measured $500 \mu \mathrm{m} \times 500 \mu \mathrm{m}$ were generated within the annotated areas. Separate image analysis protocols were developed using images, which represented the staining variation for each protein. The nuclei were detected using a Count Transform, and the optimal color ranges for the positive nuclei were specified based on hue, saturation, and value thresholds. Two different commands were used to enhance the signal to noise in the marked area. Holes in the segmentation of a cell may occur due to color variation in the immune stain of the cell, and could exclude a positive cell if the segmented area was too small. A more accurate score was achieved using the "hole 


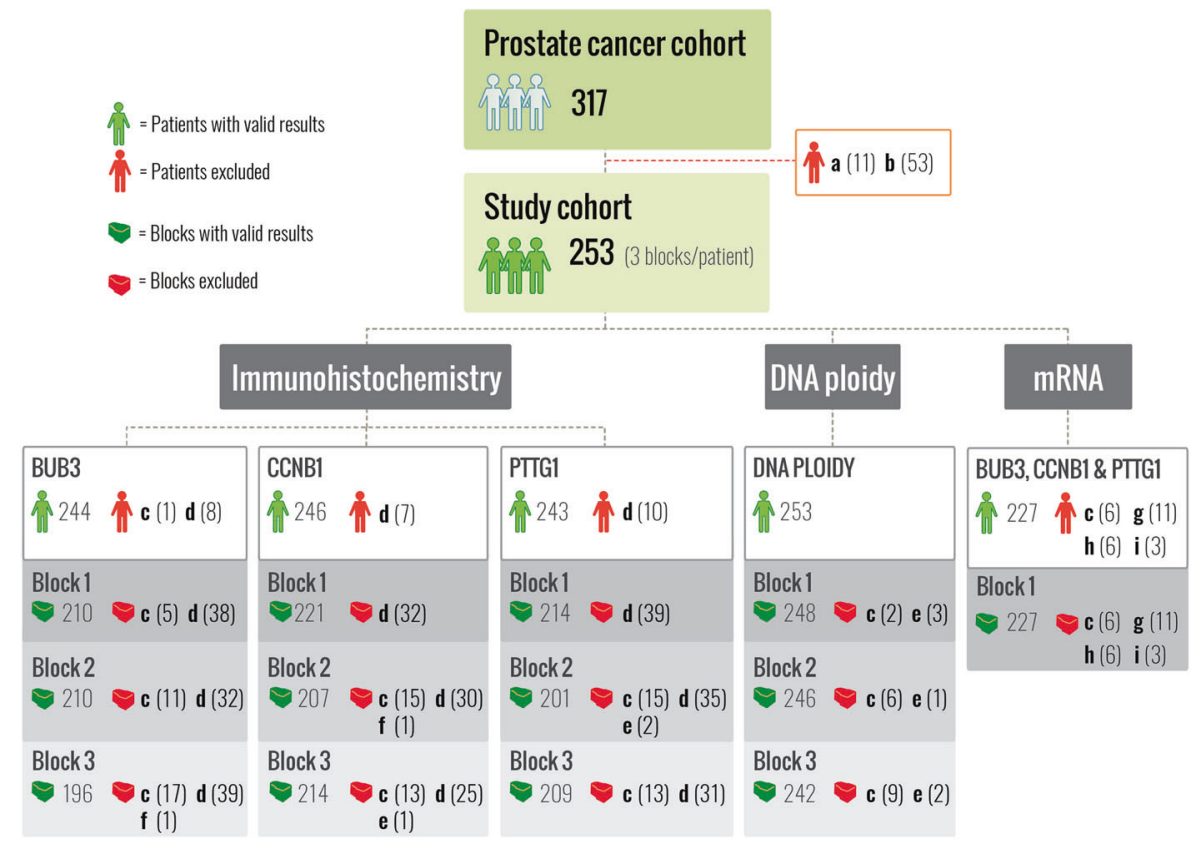

Fig. 1 Overview of patients and methods. Immunohistochemical analyses of BUB3, cyclin B1 (CCNB1) and pituitary tumortransforming 1 (PTTG1) and DNA ploidy analyses were performed on tissue sections cut from three separate tumor containing blocks. We included tumor areas $\geq 4 \mathrm{~mm}^{2}$, as measured on the diagnostic hematoxylin and eosin tissue sections. Some of the tissue blocks had been sectioned for other studies before they were included in this study. Consequently, some tumor areas were reduced to $<4 \mathrm{~mm}^{2}$ and 53 patients were excluded due to insufficient material fulfilling the inclusion criteria. The mRNA counts were analyzed with the NanoString technology in one sample corresponding to block 1 used in the immunohistochemistry study. The green patients/blocks represent the number of patients/samples with valid results, whereas the red ones represent excluded patients/samples. Exclusion criteria: ${ }^{\mathrm{a} P a t i e n t s}$ missing clinical data. ${ }^{b}$ Not fulfilling the selection criteria: three tumor containing tissue blocks were selected based on highest Gleason score

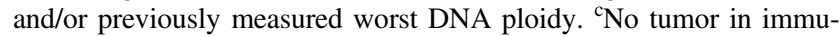
nostained section or hematoxylin and eosin control section for DNA ploidy. ${ }^{d} \geq 95 \%$ of tumor area fell off during the immunohistochemistry procedure. ${ }^{\mathrm{e}}$ Other technical issues. ${ }^{\mathrm{f}}$ Missing. ${ }^{\mathrm{g}}$ Reduced Quality Control parameters detected by nSolver. ${ }^{\mathrm{h}}>40 \%$ infiltrating benign glands.

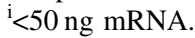

fill" function, which adds segmentation pixels to the hole. The "median $3 \times 3$ " function was used to replace each segmented pixel with the median of the surrounding segmented pixels in a 3 by 3 window. This function removes small and unspecific segmentations and fills in missing pixels in a segmented object. Furthermore, segmented objects that did not represent positively stained cells were excluded by removing both small ( $<100$ pixels) and large (>2000 pixels) objects (Supplementary Table 2). The number of positive cells and the size of the tumor area were reported by ImmunoPath and positive cells $/ \mathrm{mm}^{2}$ was calculated.

\section{DNA ploidy and RNA analyses}

DNA ploidy was analyzed by image cytometry as previously described [1,5]. The samples were classified as diploid or non-diploid (tetraploid and aneuploid). Quantification of RNA was performed on 297 tumor areas from one tissue block from the 253 patients as described in supplementary methods.

\section{Thresholds}

In the subsequent analyses, the automatic scores of CCNB1 and PTTG1 were dichotomized by the 75 th percentile as described in Supplementary Fig. 1, whereas the mRNA counts of BUB3 were dichotomized by the 25 th percentile as described in Supplementary Fig. 2.

\section{Statistical analysis}

Time to recurrence, defined in accordance with Punt and coworkers [12], was the primary endpoint and was calculated from surgery to recurrence of disease or 31.12.2008. Recurrence rates were compared with the SPSS software (v23.0, IBM Corporation, Armonk, NY) using the Mantel-Cox log-rank test in multivariable analysis of categorical variables and the Wald chi-squared test in univariable analysis of continuous variables and in multivariable analyses. Concordance index (c-index) [13] with bias-corrected and accelerated confidence interval (CI) over 10000 bootstraps [14] was computed in Stata/SE 15.1 
(StataCorp, College Station, TX). Two-sided $p$ value for test of difference in c-index was calculated as 1 minus the confidence level of the largest bias-corrected and accelerated CI that did not contain 0 . Two-sided $p$ values $<0.05$ were considered statistical significant.

\section{Results}

\section{Patients}

The patients were followed as described by Wæhre et al. [15] for a median of 11.1 (interquartile range (IQR) 7.5-14.2) years and recurrence was observed in $86 / 253$ (34\%) patients. Local recurrence was most frequently confirmed by biopsy, alternatively by palpation and/or ultrasound, whereas metastases were confirmed by scintigraphy. Local recurrence was observed in 36 patients, metastases were confirmed in 22 patients, and 23 patients had both local recurrence and metastases. The remaining five patients died of prostate cancer according to their death certificate. An overview of clinicopathological data and included samples are presented in Table 1 and Fig. 1.

\section{Protein}

BUB3 ( $n=244,616$ samples) was expressed in the nuclei of both benign epithelial and tumor cells. Additional cytoplasmic stain was observed in a subset of the tumor cells (Fig. 2). The inter-observer agreement of the cytoplasmic BUB3 score was substantial (Cohen's $\kappa=0.71$, 92\% with equal score). We observed 78 (32\%) patients with $\geq 1$ cytoplasmic BUB3 positive tumor area and $126(52 \%)$ patients with $\geq 1$ tumor area with decreased levels of nuclear BUB3. Cytoplasmic and nuclear stain of CCNB1 $(n=$ 246,642 samples) and PTTG1 ( $n=243,624$ samples) were observed in a low fraction of the tumor cells (Fig. 2). The median positive cells $/ \mathrm{mm}^{2}$ was 31 (IQR 18-55) for CCNB1 and 24 (IQR 14-40) for PTTG1 (Supplementary Fig. 1). Positive scores in $\geq 1$ tumor area were observed for 94 (38\%) patients for CCNB1 and 103 (42\%) patients for PTTG1. The categorized scores of CCNB1 and PTTG1 $(\mathrm{n}=238)$ were significantly correlated (Pearson's $r=0.60$, $p<0.001,81 \%$ with equivalent scores).

\section{mRNA}

We observed a median of 67 (IQR 52-81) BUB3 transcripts and decreased counts of BUB3 mRNA in 56 patients $(n=227$, Fig. 1). The median expression was 11 (IQR 7-15) mRNA transcripts for CCNB1 and 4 (IQR 2-5) for PTTG1, and these counts were considered below the detection level that can be expected to provide reliable estimation of mRNA expression.

\section{DNA ploidy}

We observed $111(44 \%)$ patients with $\geq 1$ tumor area with non-diploid DNA ploidy classification. DNA ploidy status was weakly correlated to the dichotomized scores of cytoplasmic BUB3 and CCNB1, but not to the PTTG1 scores (Supplementary Tables 3 and 4).

\section{Survival analyses}

Prognostic value was observed for the dichotomized scores of cytoplasmic BUB3, CCNB1, and PTTG1 in both univariable (Fig. 3) and multivariable analyses (Supplementary Table 5). DNA ploidy status was significant in univariable analysis only (hazard ratio $[\mathrm{HR}]=2.33,95 \%$ CI $1.51-3.60$, $p<0.001$ ), whereas nuclear BUB3 (Fig. 3) and BUB3 mRNA counts (Supplementary Fig. 2) were not significant.

\section{Combined risk assessment}

When including cytoplasmic BUB3, CCNB1, and PTTG1 scores in a multivariable model with other clinicopathological variables, cytoplasmic BUB3 $(\mathrm{HR}=2.29$, 95\% CI 1.39-3.80) and CCNB1 (HR $=2.2895 \%$ CI 1.19-4.38) remained significant, while PTTG1 (HR $=1.08$, 95\% CI 0.56-2.10) did not (Supplementary Table 6). When combining the markers of cytoplasmic BUB3 and CCNB1, intermediate risk of recurrence was observed for patients with tumors positive for only one of the proteins (univariable $\mathrm{HR}=2.50,95 \% \mathrm{CI} 1.46-4.27$, multivariable $\mathrm{HR}=$ $2.55,95 \%$ CI 1.44-4.53) and high risk for patients with tumors positive for both proteins (univariable $\mathrm{HR}=5.78$, 95\% CI 3.18-10.52, multivariable $\mathrm{HR}=4.25$, 95\% CI 2.11-8.53), compared with the low risk observed for patients with negative scores of both proteins (Fig. 4 and Table 2). CCNB1 and cytoplasmic BUB3 scores were integrated with the Cancer of the Prostate Risk assessment post-Surgical (CAPRA-S) score by adding two points for each marker if they were positive. The c-index of the modified CAPRA-S risk score was $0.78 \quad(95 \%$ CI 0.73-0.83), compared with 0.76 (95\% CI, 0.70-0.81) for the standard CAPRA-S. The observed difference of 0.02 between these c-indices was significant $(95 \%$ CI $0.001-0.05, p=0.044)$.

\section{Heterogeneity}

A tumor, with at least two valid results, was considered heterogeneous when positive and negative scores were observed in different tumor areas from the same tumor. The percentage of patients with heterogeneous tumors (Table 3) was estimated for cytoplasmic BUB3 (30\%), nuclear BUB3 (48\%), CCNB1 (28\%), and PTTG1 (35\%). Patients with 
Table 1 Clinicopathological data.

\begin{tabular}{|c|c|c|c|c|}
\hline Variable & $n$ & No recurrence, $n$ & Recurrence, $n$ & $p$ value $^{\mathrm{a}}$ \\
\hline Study Cohort & 253 & 167 & 86 & \\
\hline Age at surgery & & & & 0.642 \\
\hline Median (IQR) & $62(58-67)$ & $62(58-67)$ & $63(58-67)$ & \\
\hline Age at surgery & & & & 0.769 \\
\hline$\leq 65$ & 162 & 108 & 54 & \\
\hline$>65$ & 91 & 59 & 32 & \\
\hline Preoperative PSA (ng/ml) & & & & $<0.001$ \\
\hline$\leq 6$ & 57 & 49 & 8 & \\
\hline$>6$ and $\leq 10$ & 48 & 41 & 7 & \\
\hline$>10$ and $\leq 20$ & 84 & 48 & 36 & \\
\hline$>20$ & 62 & 29 & 33 & \\
\hline Missing & 2 & 0 & 2 & \\
\hline Gleason score & & & & $<0.001$ \\
\hline$\leq 6$ & 11 & 11 & 0 & \\
\hline $3+4$ & 92 & 83 & 9 & \\
\hline $4+3$ & 77 & 48 & 29 & \\
\hline$\geq 8$ & 73 & 25 & 48 & \\
\hline Surgical margins & & & & 0.001 \\
\hline Negative & 91 & 72 & 19 & \\
\hline Positive & 162 & 95 & 67 & \\
\hline Extracapsular extension & & & & $<0.001$ \\
\hline Absent & 55 & 50 & 5 & \\
\hline Present & 196 & 115 & 81 & \\
\hline Missing & 2 & 2 & 0 & \\
\hline Seminal vesicle invasion & & & & $<0.001$ \\
\hline Absent & 187 & 144 & 43 & \\
\hline Present & 66 & 23 & 43 & \\
\hline Lymph node invasion & & & & 0.014 \\
\hline Absent & 239 & 162 & 77 & \\
\hline Present & 14 & 5 & 9 & \\
\hline CAPRA-S risk group & & & & $<0.001$ \\
\hline Low $(0-2)$ & 30 & 30 & 0 & \\
\hline Intermediate (3-5) & 87 & 75 & 12 & \\
\hline High (6-12) & 132 & 60 & 72 & \\
\hline Missing & 4 & 2 & 2 & \\
\hline DNA ploidy & & & & $<0.001$ \\
\hline Diploid & 142 & 109 & 33 & \\
\hline Tetraploid & 67 & 34 & 33 & \\
\hline Aneuploid & 44 & 24 & 20 & \\
\hline Follow-up, years & & & & 0.047 \\
\hline Median (IQR) & $11.1(7.5-14.2)$ & $10.1(7.3-14.1)$ & $12.3(9.1-14.3)$ & \\
\hline
\end{tabular}

$I Q R$ inter quartile range

aAssociations were evaluated using the Pearson's $\chi^{2}$ test for categorical variables, Kendall's $\tau$ test for ordinal variables and Mann-Whitney's $U$-test for continuous variables

homogenous negative tumors of cytoplasmic BUB3, CCNB1 or PTTG1, or homogenous positive tumors of nuclear BUB3 were at lower risk of recurrence compared with patients with heterogeneous tumors. The highest risk of recurrence was observed for the patients with homogenous positive tumors of cytoplasmic BUB3, CCNB1 or 

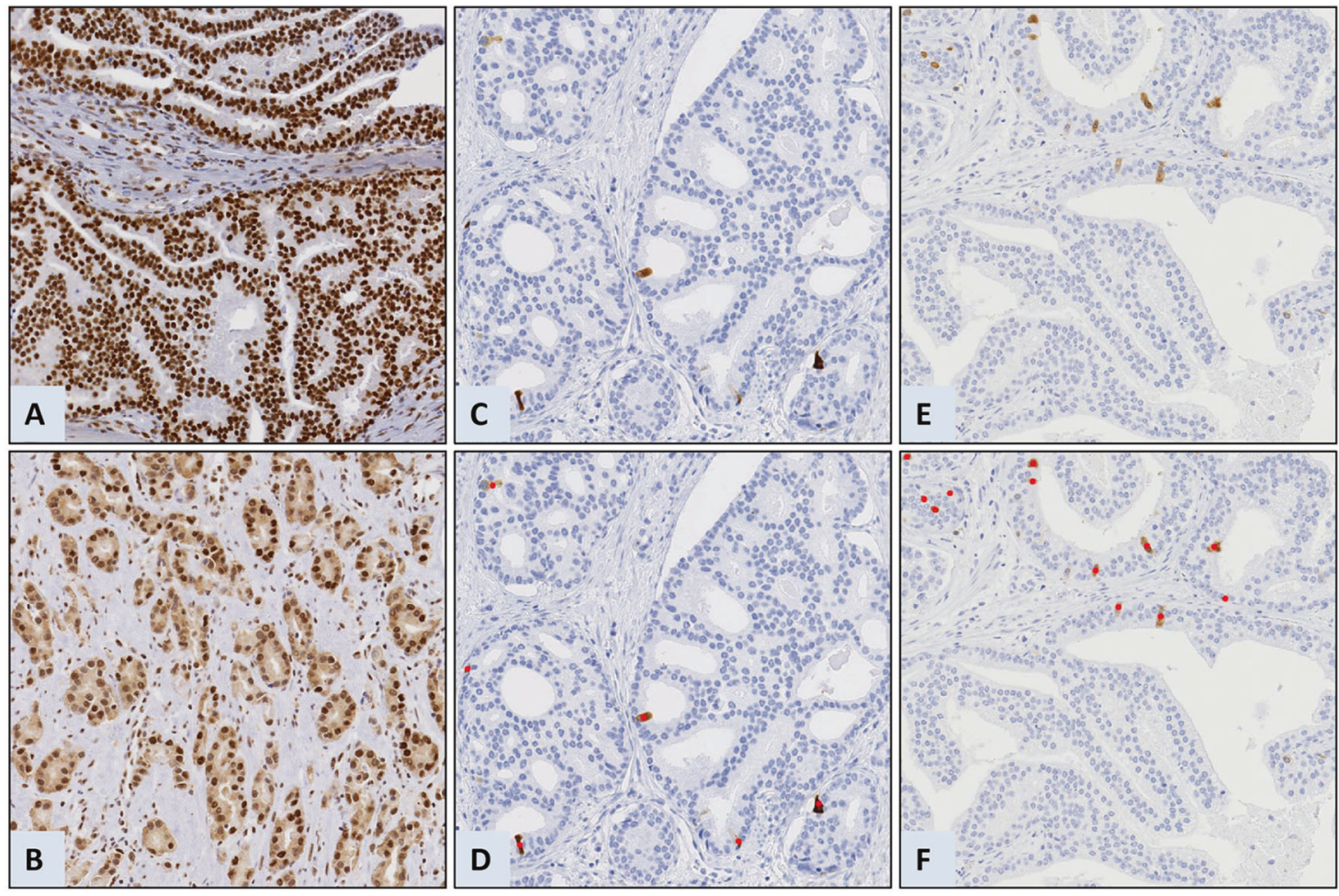

Fig. 2 Examples of protein labeling and scoring in tissue samples from prostate cancer. Nuclear and cytoplasmic protein levels of mitotic checkpoint protein BUB3, cyclin B1 (CCNB1) and pituitary tumor-transforming 1 (PTTG1) was scored by assessing the whole tumor area. The tiles represent examples of (a) nuclear BUB3,

PTTG1, or tumors with homogenous decreased expression of nuclear BUB3 (Fig. 3 and Table 3).

\section{Discussion}

The present study is the largest published study on the prognostic value of BUB3, CCNB1, and PTTG1 expression in prostate cancer. We observed independent prognostic value of cytoplasmic BUB3 and CCNB1 when adjusted for PTTG1 and clinicopathological variables in multivariable analysis. Importantly, the highest risk of recurrence was observed for the combination of cytoplasmic BUB3 and CCNB1, which added moderately and significantly to the overall risk prediction, based on the CAPRA-S score.

BUB3 is constitutively expressed and normally restricted to the nucleus [16] where it acts as a mitotic checkpoint protein. Correspondingly, we observed that most interphase cells stained positive for nuclear BUB3. In our study, counts of mRNA was weakly associated with protein levels of nuclear BUB3, neither were associated with recurrence. The lack of significant results may be explained by the fact that only one block was included in the analysis of mRNA, as significant results were obtained for nuclear BUB3 in the analysis that considered intra-tumor heterogeneity. This (b) cytoplasmic and nuclear BUB3, (c) CCNB1, and (e) PTTG1 protein levels. Cells identified by ImmunoPath as positive are marked in red for (d) CCNB1 and (f) PTTG1. Negative cells are apparent by the blue counterstain of the nuclei. Each tile is $500 \mu \mathrm{m} \times 500 \mu \mathrm{m}$.

analysis included two or three tumor samples from each patient and demonstrated that patients with decreased levels of nuclear BUB3 in all analyzed samples were at increased risk of recurrence. Zhu and colleagues [16] identified a nuclear localization signal in the amino acid sequence of BUB3 in HeLa cells. A mutation in the nuclear localization signal of BUB3 resulted in both cytoplasmic retention and nuclear expression and reduced the ability of BUB3 to arrest cells in mitosis [16]. We observed cytoplasmic expression of BUB3 in $32 \%$ of the cases and the presence of BUB3 in the cytoplasm indicated disease recurrence. The BUB3 pre-absorbed with blocking peptides was nonreactive in the tested samples, attesting to the specificity and validity of the observed cytoplasmic staining.

CCNB1 and PTTG1 expression is restricted to cells undergoing cell division $[17,18]$. The low expression of CCNB1 and PTTG1 observed in this study is in agreement with the low proliferation rates in prostate cancer [19] and comparable to the protein levels found in small-scale studies in prostate cancer $[20,21]$. The expression pattern of CCNB1 and PTTG1 is expected to be correlated as the degradation of both proteins is regulated by the mitotic checkpoint and completed before the mitotic exit $[17,18]$. In our study, the categorized expression of the two genes was significantly correlated with equivalent scores in $81 \%$ 

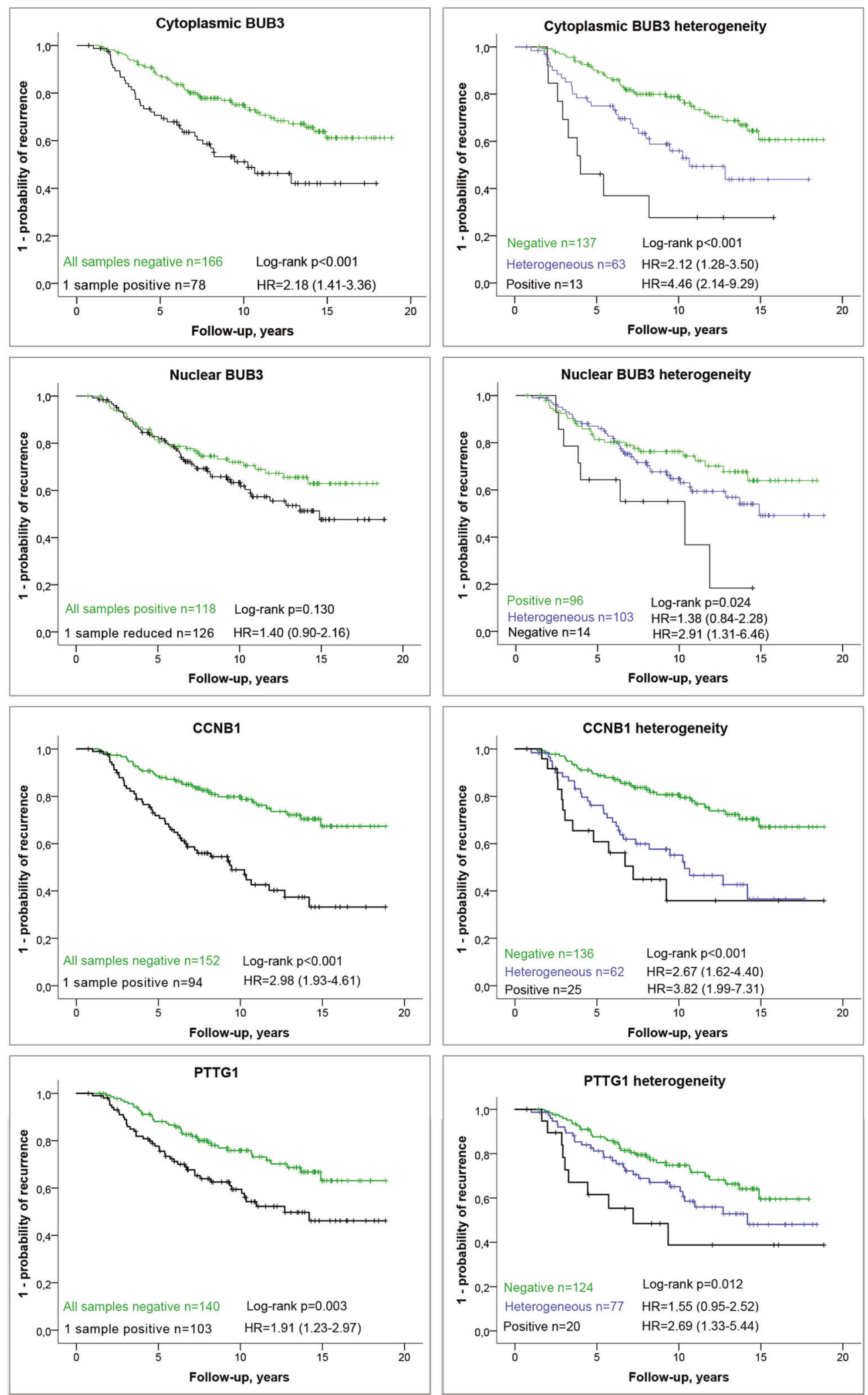

of the tumors. However, we observed a higher median expression of CCNB1 compared with PTTG1 which contradicts the reported longer half-lives of PTTG1 when compared with CCNB1 [22]. The overexpression of CCNB1 may be contributed to either unscheduled expression of CCNB1 in $\mathrm{G}_{1}$ phase [23] or sustained expression of 
Fig. 3 Univariable results of recurrence for cytoplasmic BUB3, nuclear BUB3, cyclin B1 (CCNB1), and pituitary tumortransforming 1 (PTTG1). Left panel: A tumor was considered positive for cytoplasmic BUB3 $(n=244)$, decreased of nuclear BUB3 $(n=244)$, CCNB1 positive $(n=246)$, or PTTG1 positive $(n=243)$, if this score was observed in at least one of the three measured tumor areas. The threshold for a positve score was set to $\geq 5 \%$ positivity for cytoplasmic BUB3, 55 positive cells $/ \mathrm{mm}^{2}$ for CCNB1 and 40 positive cells $/ \mathrm{mm}^{2}$ for PTTG1. The threshold for decreased nuclear BUB3 was set to $<99 \%$ positivity. Right panel: all patients with at least two valid results were included in analyses of heterogeneity of cytoplasmic BUB3 $(n=213)$, nuclear BUB3 $(n=213)$, CCNB1 $(n=223)$, or PTTG1 $(n=221)$. A tumor was considered heterogeneous when positive and negative tumor samples were observed in different tumor areas analyzed from the same tumor. Recurrence was defined as locoregional recurrence, metastasis or death from prostate cancer. The 95\% confidence intervals of the hazard ratio (HR) are listed in parentheses.

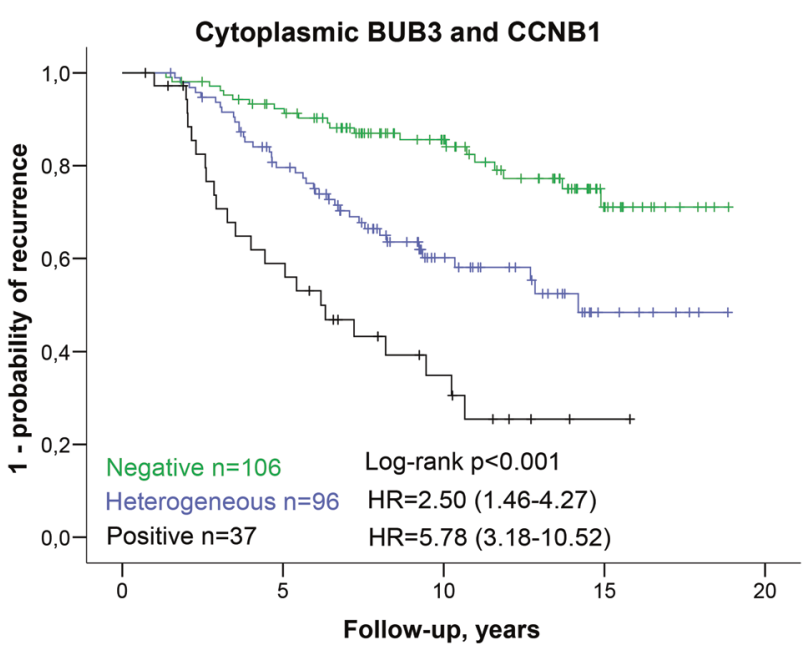

Fig. 4 Univariable results of recurrence related to cytoplasmic BUB3 scores combined with cyclin B1 (CCNB1) scores. Valid results for both cytoplasmic BUB3 and CCNB1 were obtained for 239 patients. A tumor was considered positive or negative when a tumor was positive or negative for both of the proteins. Heterogeneous scores, where the tumors were positive for either cytoplasmic BUB3 or CCNB1, was observed in 96 patients. Recurrence was defined as locoregional recurrence, metastasis, or death from prostate cancer. The 95\% confidence intervals of the hazard ratio (HR) are listed in parentheses.

CCNB1 as a consequence of $\mathrm{G}_{2}$ arrest [24]. Upregulation of PTTG1 was shown in a study describing a gene-expression signature connected to metastasis in solid tumors, including prostate cancer [25], and underscores the potential usefulness of this gene in prostate cancer prognostication. However, only CCNB1 remained significant when both proteins were included in a multivariable analysis.

Deregulation of genes involved in the mitotic checkpoint, such as BUB3 [26], CCNB1 [27], and PTTG1 [28], can contribute to cancer development by increasing the risk of incorrect cell division, aneuploidy, and genomic instability [29]. We observed that DNA ploidy was weakly associated to
Table 2 Multivariable analysis (Cox-regression) of cytoplasmic BUB3 and cyclin B1 (CCNB1) scores combined.

\begin{tabular}{|c|c|c|c|}
\hline Variable & HR & $95 \% \mathrm{CI}$ & $p$ value \\
\hline \multicolumn{3}{|l|}{ Cytoplasmic BUB3 \& CCNB1 } & $<0.001$ \\
\hline Negative & Ref & & \\
\hline Heterogeneous & 2.55 & $1.44-4.53$ & 0.001 \\
\hline Positive & 4.25 & $2.11-8.53$ & $<0.001$ \\
\hline \multicolumn{3}{|l|}{ Gleason score } & $<0.001$ \\
\hline $3+3$ & NA & NA & NA \\
\hline $3+4$ & Ref & & \\
\hline $4+3$ & 2.88 & $1.10-7.51$ & 0.031 \\
\hline$\geq 8$ & 6.49 & $2.57-16.38$ & $<0.001$ \\
\hline Seminal vesicle invasion & 1.52 & $0.91-2.53$ & 0.108 \\
\hline Extracapsular extension & 0.87 & $0.32-2.40$ & 0.788 \\
\hline Lymph node invasion & 1.99 & $0.90-4.39$ & 0.090 \\
\hline Surgical margins & 1.16 & $0.61-2.20$ & 0.659 \\
\hline \multicolumn{3}{|l|}{ Preoperative PSA $^{\mathrm{a}}$} & $0.147^{\mathrm{b}}$ \\
\hline$\leq 6$ & Ref & & \\
\hline$>6$ and $\leq 10$ & 1.19 & $0.39-3.63$ & 0.764 \\
\hline$>10$ and $\leq 20$ & 2.27 & $0.97-5.31$ & 0.058 \\
\hline$>20$ & 2.28 & $0.96-5.38$ & 0.061 \\
\hline $\mathrm{Age}^{\mathrm{c}}$ & 0.96 & $0.92-0.99$ & 0.022 \\
\hline \multicolumn{4}{|c|}{$\begin{array}{l}C I \text { confidence interval, } H R \text { Hazard ratio, } N A \text { not available } \\
{ }^{a} \mathrm{ng} / \mathrm{ml}\end{array}$} \\
\hline \multicolumn{4}{|c|}{$\begin{array}{l}{ }^{\mathrm{b}} \text { Preoperative PSA was a significant marker of recurrence when } \\
\text { included as a continuous variable in a cox regression model: } \mathrm{HR}= \\
1.02,95 \% \text { CI } 1.00-1.03, p=0.031\end{array}$} \\
\hline
\end{tabular}

cytoplasmic BUB3 and CCNB1 expression. As multiple genes and processes contribute to genomic instability [30], an association should not necessarily be expected when only a few of them are studied, particularly in samples collected at a single time-point given by surgery date. Furthermore, studies in cell lines suggests that cancer cells depend on a functional mitotic checkpoint [4] and increased expression of CCNB1 and PTTG1 is therefore more likely a result of increased proliferation. This may explain why non-diploid tumors were equally distributed in tumors with positive and negative cytoplasmic BUB3 and CCNB1 expression.

Scoring of PTTG1 and CCNB1 may prove challenging due to the low abundance of these proteins in prostate cancer. An automatic scoring system was used to overcome this challenge, allowing us to count positive tumor cells in an accurate and reproducible manner, thereby eliminating observer variability. The expression of BUB3 was only scored visually as the automatic scoring could not be applied due to technical challenges. However, the interobserver agreement was substantial. Another limitation included the use of a patient cohort from the pre-PSA era containing more aggressive tumors compared with 
Table 3 Tumor heterogeneity.

\begin{tabular}{|c|c|c|c|c|}
\hline & Block 1 & Block 2 & Block 3 & Combined $^{\mathrm{a}}$ \\
\hline \multicolumn{5}{|c|}{ Cytoplasmic BUB3 } \\
\hline$n$ & 210 & 210 & 196 & 213 \\
\hline Negative & 182 & 182 & 138 & 137 \\
\hline Heterogeneous & NA & NA & NA & 63 \\
\hline Positive & 28 & 28 & 58 & 13 \\
\hline$p$ value $^{\mathrm{b}}$ & 0.089 & $<0.001$ & 0.001 & $<0.001$ \\
\hline HR $(95 \%$ CI) & $1.71(0.91-3.21)$ & $3.12(1.83-5.30)$ & $2.29(1.41-3.73)$ & $\begin{array}{l}2.12(1.28-3.50) \\
4.46(2.14-9.29)\end{array}$ \\
\hline \multicolumn{5}{|l|}{ Nuclear BUB3 } \\
\hline$n$ & 210 & 210 & 196 & 213 \\
\hline Positive & 157 & 141 & 139 & 96 \\
\hline Heterogeneous & NA & NA & NA & 103 \\
\hline Reduced & 53 & 69 & 57 & 14 \\
\hline$p$ value $^{\mathrm{b}}$ & 0.518 & 0.018 & 0.152 & 0.024 \\
\hline HR (95\% CI) & $1.20(0.70-2.06)$ & $1.71(1.09-2.68)$ & $1.44(0.87-2.40)$ & $\begin{array}{l}1.38(0.84-2.28) \\
2.91(1.31-6.46)\end{array}$ \\
\hline \multicolumn{5}{|l|}{$\mathrm{CCNB} 1$} \\
\hline$n$ & 221 & 207 & 214 & 223 \\
\hline Negative & 152 & 157 & 173 & 136 \\
\hline Heterogeneous & NA & NA & NA & 62 \\
\hline Positive & 69 & 50 & 41 & 25 \\
\hline$p$ value $^{\mathrm{b}}$ & $<0.001$ & 0.001 & $<0.001$ & $<0.001$ \\
\hline HR (95\% CI) & $2.94(1.86-4.63)$ & $2.33(1.42-3.82)$ & $2.51(1.51-4.19)$ & $\begin{array}{l}2.67(1.62-4.40) \\
3.82(1.99-7.31)\end{array}$ \\
\hline \multicolumn{5}{|l|}{ PTTG1 } \\
\hline$n$ & 214 & 201 & 209 & 221 \\
\hline Negative & 149 & 152 & 167 & 124 \\
\hline Heterogeneous & NA & NA & NA & 77 \\
\hline Positive & 65 & 49 & 42 & 20 \\
\hline$p$ value $^{\mathrm{b}}$ & 0.036 & 0.041 & 0.014 & 0.012 \\
\hline HR $(95 \% \mathrm{CI})$ & $1.65(1.03-2.64)$ & $1.68(1.04-2.73)$ & $1.95(1.14-3.34)$ & $\begin{array}{l}1.55(0.95-2.52) \\
2.69(1.33-5.44)\end{array}$ \\
\hline
\end{tabular}

CI confidence interval, $H R$ hazard ratio, $N A$ not available

${ }^{a}$ Patients were included in the combined analyses when a patient had $\geq 2$ valid samples, a tumor was considered heterogeneous when both positive and negative scores were observed

${ }^{\mathrm{b}}$ Log-rank $p$ value

contemporary cohorts. As a consequence, the relevance of these markers should be validated in a cohort that is more representative for patients diagnosed today, including biopsy material from patients under active surveillance. However, the long follow-up gave us the advantage of using clinical recurrence as an endpoint in the survival analyses. A sampling bias caused by limited tumor material for some of the patients, may have excluded patients with a low tumor burden. Furthermore, some tumor samples were excluded as the tumor tissue fell off during the immunohistochemistry procedure. This is a well-known issue [31], which we tried to avoid by using coated slides and removing excess water in the sections before freezing.
Intra-tumor heterogeneity is a challenge for biomarker studies. The heterogeneity of gene expression of BUB3, CCNB1, and PTTG1 was assessed at the protein level, and observed in about one third of the tumors. The prognostic value improved when we combined the results from three tissue blocks from a patient into one score for each of the proteins. This is in line with the previously described impact of heterogeneity on DNA ploidy as a prognostic marker [1]. Our results indicate that it is possible to account for intratumor heterogeneity by analysis of multiple tumor samples, which is a disadvantage in a clinical setting. On the other hand, univariable analyzes of the separate series of tumor samples were statistically significant, which means that one 
tissue block per patient may be sufficient to assess CCNB1 and PTTG1 expression. Significant results were only obtained for two of the series for cytoplasmic BUB3 and one series for nuclear BUB3, which necessitates analyses of multiple tumor samples for this protein. However, our threetier system indicates that the presence of intra-tumor heterogeneity is a marker of prognostic importance in itself. We would therefore recommend that the evaluation of these proteins should be examined in more than one tissue block for each patient.

\section{Conclusion}

The prognostic value of cytoplasmic BUB3 expression in prostate carcinomas has been reported for the first time in this study. The positive expression of cytoplasmic BUB3, CCNB1, and PTTG1 was significantly correlated with recurrence. Evaluation of the investigated proteins in three tissue blocks considerably improved their prognostic value. The combination of cytoplasmic BUB3 and CCNB1 stratified the patients into three risk groups with a 10 -year recurrence rate of $16 \%$ for the low-risk group, $40 \%$ for the intermediate risk group and $65 \%$ for the high-risk group. This marker has the potential to enable better risk stratification of patients with prostate cancer, as it significantly improved the CAPRA-S risk stratification tool.

Acknowledgements We thank Marna Lill Kjæreng, Maria X. Isaksen and Marte S. Linjordet for technical assistance, and Hans Martin Mohn for assistance with ImmunoPath. This work was supported by the South-Eastern Norway Regional Health Authority research fund (grant numbers 2012025 to EE and 2013133 to KC); and by the Research Council of Norway, through its IKTPLUSS Lighthouse program (grant number 259204).

\section{Compliance with ethical standards}

Conflict of interest The authors declare that they have no conflict of interest.

Publisher's note Springer Nature remains neutral with regard to jurisdictional claims in published maps and institutional affiliations.

Open Access This article is licensed under a Creative Commons Attribution 4.0 International License, which permits use, sharing, adaptation, distribution and reproduction in any medium or format, as long as you give appropriate credit to the original author(s) and the source, provide a link to the Creative Commons license, and indicate if changes were made. The images or other third party material in this article are included in the article's Creative Commons license, unless indicated otherwise in a credit line to the material. If material is not included in the article's Creative Commons license and your intended use is not permitted by statutory regulation or exceeds the permitted use, you will need to obtain permission directly from the copyright holder. To view a copy of this license, visit http://creativecommons. org/licenses/by/4.0/.

\section{References}

1. Cyll K, Ersvær E, Vlatkovic L, Pradhan M, Kildal W, Kjær MA, et al. Tumour heterogeneity poses a significant challenge to cancer biomarker research. Br J Cancer. 2017;117:367-75.

2. McGranahan N, Burrell RA, Endesfelder D, Novelli MR, Swanton C. Cancer chromosomal instability: therapeutic and diagnostic challenges. EMBO Rep. 2012;13:528-38.

3. Lara-Gonzalez P, Westhorpe FG, Taylor SS. The spindle assembly checkpoint. Curr Biol. 2012;22:R966-80.

4. Kops GJ, Weaver BA, Cleveland DW. On the road to cancer: aneuploidy and the mitotic checkpoint. Nat Rev Cancer. 2005;5:773-85.

5. Danielsen HE, Pradhan M, Novelli M. Revisiting tumour aneuploidy - the place of ploidy assessment in the molecular era. Nat Rev Clin Oncol. 2016;13:291-304.

6. Bie L, Zhao G, Cheng P, Rondeau G, Porwollik S, Ju Y, et al. The accuracy of survival time prediction for patients with glioma is improved by measuring mitotic spindle checkpoint gene expression. PLoS ONE. 2011;6:e25631.

7. Genkai N, Homma J, Sano M, Tanaka R, Yamanaka R. Increased expression of pituitary tumor-transforming gene (PTTG)- 1 is correlated with poor prognosis in glioma patients. Onco Rep. 2006;15:1569.

8. Sun X, Zhangyuan G, Shi L, Wang Y, Sun B, Ding Q. Prognostic and clinicopathological significance of cyclin $\mathrm{B}$ expression in patients with breast cancer A meta-analysis. Med (Baltim). 2017;96:e6860.

9. Attard G, Parker C, Eeles RA, Schröder F, Tomlins SA, Tannock I, et al. Prostate cancer. Lancet. 2016;387:70-82.

10. McShane LM, Altman DG, Sauerbrei W, Taube SE, Gion M, Clark GM. REporting recommendations for tumour MARKer prognostic studies (REMARK). Br J Cancer. 2005;93:387-91.

11. Epstein JI. An update of the Gleason grading system. J Urol. 2010;183:433-40.

12. Punt CJ, Buyse M, Köhne $\mathrm{CH}$, Hohenberger $\mathrm{P}$, Labianca R, Schmoll HJ, et al. Endpoints in adjuvant treatment trials: a systematic review of the literature in colon cancer and proposed definitions for future trials. J Natl Cancer Inst. 2007;99:998-1003.

13. Harrell FE Jr., Califf RM, Pryor DB, Lee KL, Rosati RA. Evaluating the yield of medical tests. JAMA. 1982;247:2543-6.

14. Efron B. Better Bootstrap Confidence Intervals. J Am Stat Assoc. 1987;82:171-85.

15. Wæhre H, Vlatkovic L, Cvancarova M, Paus E, Fosså SD, Danielsen HE. Fifteen-year mortality after radical prostatectomy: which factors are available for patient counselling? Scand J Urol. 2014;48:123-30.

16. Zhu S, Jing R, Yang Y, Huang Y, Wang X, Leng Y, et al. A Motif from $\mathrm{Lys}^{216}$ to Lys ${ }^{222}$ in human BUB3 protein is a nuclear localization signal and critical for BUB3 function in mitotic checkpoint. J Biol Chem. 2015;290:11282-92.

17. Pines J, Hunter T. Human cyclins A and B1 are differentially located in the cell and undergo cell cycle-dependent nuclear transport. J Cell Biol. 1991;115:1-17.

18. Vlotides G, Cruz-Soto M, Rubinek T, Eigler T, Auernhammer CJ, Melmed S. Mechanisms for growth factor-induced pituitary tumor transforming gene-1 expression in pituitary folliculostellate $\mathrm{TtT} /$ GF cells. Mol Endocrinol. 2006;20:3321-35.

19. Desmeules P, Hovington H, Nguilé-Makao M, Léger C, Caron A, Lacombe L, et al. Comparison of digital image analysis and visual scoring of KI-67 in prostate cancer prognosis after prostatectomy. Diagn Pathol. 2015;10:67.

20. Mashal RD, Lester S, Corless C, Richie JP, Chandra R, Propert $\mathrm{KJ}$, et al. Expression of cell cycle-regulated proteins in prostate cancer. Cancer Res. 1996;56:4159-63. 
21. Zhu X, Mao Z, Na Y, Guo Y, Wang X, Xin D. Significance of pituitary tumor transforming gene 1 (PTTG1) in prostate cancer. Anticancer Res. 2006;26:1253-9.

22. Sharova LV, Sharov AA, Nedorezov T, Piao Y, Shaik N, Ko MS. Database for mRNA half-life of 19977 genes obtained by DNA microarray analysis of pluripotent and differentiating mouse embryonic stem cells. DNA Res. 2009;16: $45-58$.

23. Gorczyca W, Sarode V, Juan G, Melamed MR, Darzynkiewicz Z. Laser scanning cytometric analysis of cyclin B1 in primary human malignancies. Mod Pathol. 1997;10:457-62.

24. Chang DC, Xu N, Luo KQ. Degradation of cyclin B is required for the onset of anaphase in Mammalian cells. J Biol Chem. 2003;278:37865-73.

25. Ramaswamy S, Ross KN, Lander ES, Golub TR. A molecular signature of metastasis in primary solid tumors. Nat Genet. 2003;33:49-54.

26. Babu JR, Jeganathan KB, Baker DJ, Wu X, Kang-Decker N, van Deursen JM. Rae1 is an essential mitotic checkpoint regulator that cooperates with Bub3 to prevent chromosome missegregation. J Cell Biol. 2003;160:341-53.

27. Kallakury BV, Sheehan CE, Rhee SJ, Fisher HA, Kaufman RP Jr., Rifkin MD, et al. The prognostic significance of proliferationassociated nucleolar protein p120 expression in prostate adenocarcinoma: a comparison with cyclins A and B1, Ki-67, proliferating cell nuclear antigen, and $\mathrm{p} 34^{\mathrm{cdc} 2}$. Cancer. 1999;85:1569-76.

28. Yu R, Lu W, Chen J, McCabe CJ, Melmed S. Overexpressed pituitary tumor-transforming gene causes aneuploidy in live human cells. Endocrinology. 2003;144:4991-8.

29. Funk LC, Zasadil LM, Weaver BA. Living in CIN: mitotic infidelity and its consequences for tumor promotion and suppression. Dev Cell. 2016;39:638-52.

30. Carter SL, Eklund AC, Kohane IS, Harris LN, Szallasi Z. A signature of chromosomal instability inferred from gene expression profiles predicts clinical outcome in multiple human cancers. Nat Genet. 2006;38:1043-8.

31. Kim SW, Roh J, Park CS. Immunohistochemistry for pathologists: protocols, pitfalls, and tips. J Pathol Transl Med. 2016;50:411-8. 